\title{
Adherence to Antihypertensive Medications and Some of Its Clinical Implications in Patients Seen At a Tertiary Hospital in Nigeria
}

\author{
Ebenezer Adekunle Ajayi ${ }^{1}$, Adekunle Olatayo Adeoti ${ }^{1}$, Iyiade Adeseye Ajayi ${ }^{2}$, \\ Akande Oladimeji Ajayi ${ }^{1}$, Victor O. Adeyeye ${ }^{3}$ \\ ${ }^{1}$ Department of Medicine, Ekiti State University Teaching Hospital, Ado Ekiti, Nigeria \\ ${ }^{2}$ Department of Ophthalmology, Ekiti State University Teaching Hospital, Ado Ekiti, Nigeria \\ ${ }^{3}$ Department of Medicine, Obafemi Awolowo University Teaching Hospital Complex, Ile-ife, Nigeria
}

\begin{abstract}
:
Background: Adherence to antihypertensive is crucial to treatment success in subjects with hypertension. This study is aimed at assessing antihypertensive drug adherence using validated tool and its relationship with some demographic and clinical parameters in outpatient hypertensive subjects.

Methods:The Morisky Medication Adherence Scale (MMAS-4) was used to assess drug adherence status in 150 hypertensive patients who were consecutively recruited in a Medical Outpatient Department of a Tertiary Health Centre. All the patients had resting 12-lead electrocardiogram (ECG) done to determine left ventricular hypertrophy $(\mathrm{LVH})$. Data were analyzed with SPSS 13 and statistical significance of $p<0.05$ was used.

Results: One hundred and fifty hypertensive patients with a mean age of $61.53 \pm 9.72 y e a r s$ were recruited over a period of 6 months. Sixty seven (44.7\%) were good drug adherers while 55.3\% were not. Patients with poor antihypertensive adherence had higher Sokolow- Lyon ECG score $(34.46 \pm 7.87$ vs. $22.96 \pm 7.07 ; p=0.07)$ as well as longer $P$ wave duration on ECG $(0.15 \pm 0.03$ vs. $0.12 \pm 0.04$; $p=0.03)$. There was higher rate of $E C G$ LVH among patients with poor drug adherence (47.0\%) compared to patients with good drug adherence $(4.5 \%)$. Significant correlates of poor drug adherence were age $(r=-0.221 ; p=0.022)$ and BMI $(r=0.431$; $P<0.001$ ).

Conclusion:The rate of poor drug compliance was high with preponderance of $L V H$ in poor adherer. Physicians will do well to engage their hypertensive patients on hypertension and its treatment with a view to improving drug adherence in them.
\end{abstract}

Keywords: Medication, adherence, hypertension, electrocardiogram

\section{Introduction}

Hypertension is a major health problem in both developed and developing countries and is estimated to cause more than $13 \%$ of deaths annually [1]. The combined prevalence rates of hypertension in Africa and Nigeria are $15 \%$ and $22 \%$, respectively $[2,3]$. There are various antihypertensive drugs that have been shown to effectively control blood pressure, thereby reducing the attendant risk of cardiovascular morbidity and mortality. However, poor or non adherence to antihypertensive medications has been a challenge in the care of patients with hypertension.

A World Health Organization report [4] estimates that adherence to antihypertensive medications ranges from 52\% to $74 \%$ when adherence is defined as possession of a medication at least $80 \%$ of the time. This World Health Organization report identified non-adherence to medical treatment as a major public health concern, especially in patients with chronic conditions such as hypertension [4], and a major cause of suboptimal control of blood pressure in hypertensive subjects. It is documented that uncontrolled blood pressure increases the risk of ischemic heart disease 3-to 4-fold [5] and the overall cardiovascular risk by 2-to 3-fold [6] and that non adherence to therapy is associated with an increased risk of stroke in patients with hypertension [7]. To the best of our knowledge, particularly in the study area, little is known about antihypertensive drug adherence status and its effect on the treatment outcomes such as left ventricular hypertrophy in hypertension. Therefore, we undertook this study with the aim of assessing antihypertensive drug adherence using validated tool [8] and its relationship with some demographic and clinical parameters in outpatient hypertensive subjects on follow up at a tertiary health centre in southwest Nigeria.

\section{Methods}

One hundred and fifty consented hypertensive patients on antihypertensive drugs were consecutively recruited from the Medical Outpatient Department (MOP) of a State owned Teaching Hospital in Southwest, Nigeria. This Centre is a tertiary health Centre which serves Ekiti State and its neighboring States of Ondo 
(Southwest Nigeria), Kogi (North central Nigeria) and Kwara (North central Nigeria). Referrals to the MOP are mostly received from the General Outpatient Department (GOPD) and occasionally from other Specialist Clinics of the Hospital. Patients seen in this Hospital are expected to source for their medications from the Hospital Pharmacy Department upon out- of- pocket payment of bills for such drugs, though a few who subscribed to the National Health Insurance Scheme of the Federal Government of Nigeria may have some subsidies. Demographic parameters of subjects were noted and recorded. All subjects were clinically examined to evaluate their body mass index (BMI) and blood pressure. Fasting lipid profile, estimated creatinine clearance using Cockcroft-Gault formula [9] and resting 12-lead electrocardiogram (ECG) to determine left ventricular hypertrophy using Sokolow-Lyon criteria [10] and $\mathrm{P}$ wave duration as a measure of left atrial abnormality were done. Questions were asked on duration of antihypertensive drugs usage, risk associated with poor blood pressure control and whether or not patients have ever been informed of what their target blood pressure level should be. The 4-item Morisky questionnaire (the Morisky Medication-taking Adherence Scale- MMAS-4) [8] with a high reliability and validity was used to assess drug adherence status of the patients. This questionnaire was administered by the attending clinician at the time other data as stated above were collected. The total score ranges from 0 to 4 . During analysis, a cut-off value of MMAS mean score $\geq 2$ and $<2$ were used for labeling patients as good drug adherence or poor drug adherence respectively [11]. The study was approved by the Institution's Ethics and Research Committee.

\subsection{Statistical Analysis}

Means (Standard deviations) were used to describe the distributions of continuous variables. Percentages were used to describe categorical variables. Comparisons of categorical data were performed with the use of Pearson's chi-square test. For continuous data a Student $t$-test was used to compare means. Correlation between poor adherent status and other continuous such as age, BMI, treatment duration, blood pressure lipid profile and ECG variables was performed by Spearman's rho correlation co-efficient ' $r$ '. All statistical analyses were performed with commercially available computer program SPSS 13.0 (SPSS Inc., Chicago, IL). $P<0.05$ was considered statistically significant.

\section{Results}

One hundred and fifty patients $(M=63, \mathrm{~F}=87)$ with hypertension were interviewed and examined over a period of six months. The mean age was $61.53 \pm 9.72$ (range 33- 81 years). One hundred and twelve $(74.7 \%)$ had a form of formal education (at least schooled up to primary school level) while the rest had none. Only 10 (6.7\%) had history of either currently smoking cigarette or had smoked in the past. The details of demographic and other clinical parameters are shown in [table 1].

Sixty seven (44.7\%) scored $\geq 2$ on MMAS-4 and were adjudged to have had 'good' drug adherence while $55.3 \%$ has score $<2$ and were considered to be poorly adherent with antihypertensive medications. The awareness of risk associated with poor blood pressure control was high (98\%) in our sample population with only $2.0 \%$ claimed not to have any idea of risks associated with high blood pressure; all of these were poorly adherent to antihypertensive drugs.

Most of the patients (66.0\%) had never been told by their Physicians of what their target blood pressure should be. As seen in figure 2, thirty eight $(25.3 \%)$ of the sample population had their blood pressure to target, even though, there was no statistically significant difference between good adherer and poor adherer (OR, 0.65; 95\% CI, 0.31 to $1.38 ; \mathrm{p}=0.26$ ).

As shown in [table 1], patients with poor antihypertensive adherence had higher Sokolow- Lyon ECG score ( $34.46 \pm 7.87$ vs. $22.96 \pm 7.07 ; \mathrm{p}=0.07)$ as well as longer $\mathrm{P}$ wave duration on ECG ( $0.15 \pm 0.03$ vs. $0.12 \pm$ $0.04 ; \mathrm{p}=0.03)$. There was higher rate of ECG LVH among patients with poor drug adherence (47.0\%) compared to $4.5 \%$ of patients with good drug adherence (OR, $0.02 ; 95 \% \mathrm{CI}, 0.006$ to $0.080 ; \mathrm{p}<0.001)$. In similar vein, out of the $13(8.7 \%)$ patients with serum TC/HDL-C > 3.5, 10(76.9\%) were patients with poor adherence to antihypertensive drugs.

Type II diabetes mellitus, congestive cardiac failure and arthritis were the identified co-morbid medical conditions requiring medications in the patients and the details are shown in [figure 1]. Majority of the patients (78.0\%) were on more than one antihypertensive drug. More patients with good drug adherence than those with poor drug adherence $\left(89.6 \%\right.$ vs. $\left.73.1 \% ; \mathrm{x}^{2}=6.30, \mathrm{p}=0.01\right)$ were on more than one antihypertensive medication. Significant correlates of poor drug adherence were age $(r=-0.221 ; \mathrm{p}=0.022)$ and BMI $(\mathrm{r}=0.431 ; \mathrm{P}<0.001)$.

\section{Discussion}

Adherence to antihypertensive medications as measured by MMAS-4 in our study was $44.7 \%$ which is lower than what was found in some parts of Ethiopia (64.6\%) where similar tool was employed [12]. This difference is possibly because more than half $(59.6 \%)$ of the patients in the Ethiopian study received free medical care and drugs whereas in our present study, patients had to pay for their treatment. Poor adherence to 
treatment in hypertension is the single most important reason for uncontrolled blood pressure, serious complications and wastage of health care resources [4]. It has been estimated by WHO that adherence to antihypertensive medications is poor, ranging from 52 to $74 \%$ when it is defined as possession of a medication at least $80 \%$ of the time [4]. The factors that mitigate adherence to antihypertensive therapy and implications of such behavior have been described [13]. Some of the factors are age, number of drugs prescribed and patients' knowledge of the disease and treatment, including their beliefs and practices.

In the present study, patients who were more likely to adhere poorly to their medications were younger with a significant inverse correlation between age and poor drug adherence. This finding is consistent with a number of other studies [13-15]. In our study, the awareness of the risk associated with poor blood pressure control was high, though most of the patients claimed they have not had any discussion with their Physicians on what their target blood pressure should be. The reason for this high knowledge of risk associated with poor blood pressure control may be because of the high literacy rate $(75 \%)$ of our study population. We however noted that the few who appeared ignorant of the risk associated with poor blood pressure control did adhere poorly to their medications. It is likely that good knowledge of risk associated with poor BP control may have a positive impact on practice of good medication adherence. Studies have suggested that patients who were aware of their diseases and treatments may have better adherence compared to those who did not [16], though earlier studies from some developed countries controverted this claim $[17,18]$.

Drug adherence was higher in those on more than one antihypertensive medication $(89.6 \%)$ compared with $73.1 \%$ on only one antihypertensive medications $\left(\mathrm{X}^{2}=6.30, \mathrm{p}=0.01\right)$. This finding is consistent with a number of other studies [13, 19], including a statement from the European Society of Hypertension and European Society of Cardiology guidelines released in 2007[20], though there are other reports which showed no association [21]. Apart from the assumption that patients on multiple pills could assume that the severity of their disease is high and therefore become more conscious of the need to use medications coupled with pressures from close family members, other reasons that may explain our finding is the improved drug side-effects profile associated with multiple medications because of the lower doses employed as well as the likelihood of patient's commitment to taking drugs when they need to take many of them when compared to those who need to take only one tablet who are more likely to forget to take it.

It was also observed in this study that patients with co-morbidities were more poorly adherent to antihypertensive medications. Co-morbidities can worsen patient's conditions and make them less likely to adhere to their antihypertensive drugs. Patients with chronic co-morbidities requiring additional medications could suffer from complex treatment regimens which may mitigate adherence to medications in addition to the possibility of an associated depressive illness. In contrast, poor drug adherence to antihypertensive medications may result in co-morbidities such as heart failure and stroke or worsen them as usually seen in microvascular complications of diabetes mellitus.

It has been documented from earlier studies that high adherence to antihypertensive treatment is associated with 30-38\% decreased risk of cardiovascular events compared with lower adherence [20, 22]. The benefits accruing from blood pressure lowering may partially explain the decreased risk of cardiovascular events among high adherer. In similar vein, left ventricular hypertrophy is an independent risk factor for the development of cardiovascular events [23]. In addition to the fact that patients with poor drug adherence in our study had higher mean voltages using Sokolow-Lyon criteria[10] compared to those with good adherence, the rate of LVH was also higher in poor drug adherer (47\%) compared to good adherer. Although, cardiovascular outcomes were not the focus of this study, the high prevalence of LVH in those with history of poor drug adherence portends a serious danger of unfavorable cardiovascular outcomes in them, more so that serum lipid profile was also in the unfavorable range in them as evidenced by the high TC/HDL-C ratio $>3.5$, also prevalent among the poor drug adherer in this study.

In conclusion, more than half of the study population was found to be poorly adherent to their antihypertensive treatment. Poor drug adherence is associated with increased prevalence of left ventricular hypertrophy and may portend serious negative consequences in terms of cardiovascular in hypertensive patients. Physicians will do well to engage their hypertensive patients on hypertension and its treatment with a view to improving drug adherence in them.

This study is not without its limitations. MMAS-4 which was used as the only method of measuring adherence has the disadvantage of recall bias and eliciting socially acceptable responses, hence, has the tendency to overestimate adherence level, though it is a validated and reliable tool with significant linear relation with adherence measured as a continuum. Again, this study was carried out in a tertiary health care setting with the findings not likely to be applicable to the general population. 
V. Conflict of interest

The authors have no conflict of interest.

References

[1]. World Health Organization (WHO). The world health report 2002-reducing risks, promoting healthy life. 2011. www. who.int/whr/2002/en/.

[2]. Salako LA: Hypertension in Africa and Effectiveness of Its Management with Various Classes of Antihypertensive Drugs and in Different Socio- Economic and Cultural Environments. Clin Exp Hypertens 1993, 15(6):997-1004.

[3]. Ekwunife OI and Aguwa CN. A meta analysis of prevalence rate of hypertension in Nigerian populations. J. Public Health Epidemiol. 2011, 3(13): 604-607.

[4]. World Health Organization. Chapter III Hypertension in Adherence to Long- Term Therapies-Evidence for Action. 2003. p. 27. Available: http:// www.who.int/chronic_conditions/adherencereport/en/print.html.

[5]. Berenson GS, et al. Association between multiple cardiovascular risk factors and atherosclerosis in children and young adults. The Bogalusa Heart Study. N Engl J Med. 1998, 338: 1650-1656.

[6]. Thompson DW and Furlan AJ. Clinical epidemiology of stroke. Neurol Clin. 1996, 14: 309-315.10.

[7]. Baune BT, Aljeesh YI, Bender R. The impact of non-compliance with the therapeutic regimen on the development of stroke among hypertensive men and women in Gaza, Palestine. Saudi Med J. 2004, 25(11): 1683-8.

[8]. Morisky DE, Green LW, Levine DM: Concurrent and predictive validity of a self-reported measure of medication adherence. Med Care 1986, 24(1):67-74.

[9]. Cockcroft DW, Gault MH. Prediction of creatinine clearance from serum creatinine. Nephron. 1976; 16(1): 31-41.

[10]. Sokolow M, Lyon TP. The ventricular complex in left ventricular hypertrophy as obtained by unipolar precordial and limb leads. Am Heart J. 1949; 37:161-186.

[11]. Inkster ME, Donnan PT, MacDonald TM, Sullivan FM, Fahey T: Adherence to antihypertensive medication and association with patient and practice factors. J Hum Hypertens 2006, 20(4):295-297.

[12]. Ambaw AD, Alemie GA, W/Yohannes SM and Mengesha ZB. Adherence to antihypertensive treatment and associated factors among patients on follow up at University of Gondar Hospital, Northwest Ethiopia. BMC Public Health 2012 12:282- 287.

[13]. Hashmi SK, Afridi MB, Abbas K, Sajwani RA, Saleheen D, et al (2007) Factors Associated with Adherence to Anti-Hypertensive Treatment in Pakistan. PLoS ONE 2(3): e280. doi:10.1371/journal.pone.0000280

[14]. Krousel-Wood M, Thomasa S, Muntner P, Morisky D. Medication adherence: a key factor in achieving blood pressure control and good clinical outcomes in hypertensive patients. Curr Opin Cardiol. 2004, 19: 357-362.

[15]. Caro JJ, Salas M, Speckman JL, et al. (1999) Persistence with treatment for hypertension in actual practice. CMAJ 160: 31-37.

[16]. Baune B, Aljeesh Y: The association of psychological stress and health related quality of life among patients with stroke and hypertension in Gaza Strip. Ann Gen Psychiatr 2006, 5(6).

[17]. Krousel-Wood M, Thomas S, Muntner P, Morisky D: Medication adherence: a key factor in achieving blood pressure control and good clinical outcomes in hypertensive patients. Curr Opin Cardiol 2004, 19(4):357-362.

[18]. Yiannakopoulou E, Papadopulos JS, Cokkinos DV, Mountokalakis TD: Adherence to antihypertensive treatment: a critical factor for blood pressure control. Eur J Cardiovasc Prev Rehabil 2005, 12(3):243-249.

[19]. Mazzaglia G, Ambrosioni E, Alacqua M, Filippi A, Sessa E, et al. Adherence to Antihypertensive Medications and Cardiovascular Morbidity Among Newly Diagnosed Hypertensive Patients. Circulation. 2009; 120:1598-1605.

[20]. Mancia G, De Backer G, Dominiczak A, Cifkova R, Fagard R, Germano G, Grassi G, Heagerty AM, Kjeldsen SE, Laurent S, Narkiewicz K, Ruilope L, Rynkiewicz A, Schmieder RE, Boudier HA, Zanchetti A, for the ESH-ESC Task Force on the Management of Arterial Hypertension. 2007 ESH-ESC practice guidelines for the management of arterial hypertension: ESH-ESC Task Force on the Management of Arterial Hypertension. J Hypertens. 2007; 25:1751-1762.

[21]. Schroeder K, Fahey T, Ebrahim S. How can we improve adherence to blood pressure-lowering medication in ambulatory care? Systematic review of randomized controlled trials. Arch Intern Med. 2004; 1645: 722-732.

[22]. Blood Pressure Lowering Treatment Trialists' Collaboration. Effects of different regimens to lower blood pressure on major cardiovascular events in older and younger adults: meta-analysis of randomised trials. BMJ. 2008;336:1121-1123.LVH

[23]. Bombelli M, Facchetti R, Carugo S, Madotto F, Arenare F, Quarti-Trevano F, et al. Left ventricular hypertrophy increases cardiovascular risk independently on in-offi ce or out-of office blood pressure values. J Hypertens. 2009; 27:2458 - 2564.

Table 1: Demographic and Clinical Parameters of the Study Population

\begin{tabular}{|l|l|l|l|}
\hline & $\begin{array}{l}\text { Good Drug Adherence } \\
\mathbf{n}(\mathbf{6 7})\end{array}$ & $\begin{array}{l}\text { Poor Drug Adherence } \\
\mathbf{n}(\mathbf{8 3})\end{array}$ & p Value \\
\hline Age(years) & $63.60 \pm 8.95$ & $59.96 \pm 10.04$ & 0.245 \\
\hline Sex & $\mathrm{M}=35 ; \mathrm{F}=32$ & $\mathrm{M}=28 ; \mathrm{F}=55$ & 0.017 \\
\hline Body Mass Index $\left(\mathrm{kgm}^{-2}\right)$ & $24.96 \pm 3.88$ & $29.25 \pm 4.61$ & 0.603 \\
\hline Cigarette Smoking(n) & Yes $=0 ; \mathrm{No}=67$ & Yes $=10 ; \mathrm{No}=73$ & 0.002 \\
\hline Treatment Duration(months) & $58.14 \pm 13.32$ & $64.36 \pm 6.49$ & $<0.001$ \\
\hline Systolic Blood Pressure $(\mathrm{mmHg})$ & $145.33 \pm 18.69$ & $151.96 \pm 22.52$ & 0.840 \\
\hline Diastolic Blood Pressure(mmHg) & $85.60 \pm 16.52$ & $91.17 \pm 12.50$ & 0.191 \\
\hline LDL-C $(\mathrm{mmol} / \mathrm{L})$ & $2.11 \pm 0.76$ & $1.31 \pm 0.97$ & 0.021 \\
\hline HDL-C $(\mathrm{mmol} / \mathrm{L})$ & $2.18 \pm 1.27$ & $2.03 \pm 0.71$ & 0.062 \\
\hline Tg(mmol/L) & $1.25 \pm 0.64$ & $1.26 \pm 0.38$ & 0.006 \\
\hline TC $(\mathrm{mmol} / \mathrm{L})$ & $4.60 \pm 0.77$ & $4.45 \pm 1.05$ & 0.675 \\
\hline TC/HDL Ratio & $2.49 \pm 0.74$ & $3.87 \pm 0.433$ & 0.053 \\
\hline eGFR $(\mathrm{ml} / \mathrm{min})$ & $90.76 \pm 13.35$ & $83.44 \pm 10.80$ & 0.148 \\
\hline Serum Creatinine( $\mu$ mol/L) & $0.84 \pm 0.22$ & $1.08 \pm 0.64$ & 0.182 \\
\hline ECG P wave duration(mm) & $0.13 \pm 0.33$ & $0.15 \pm 0.36$ & 0.031 \\
\hline ECG SV $1+$ RV $(\mathrm{mm})$ & $22.96 \pm 7.07$ & $34.46 \pm 7.87$ & 0.070 \\
\hline
\end{tabular}


LDL-C= Low Density Lipoprotein Cholesterol; HDL-C = High Density Lipoprotein Cholesterol; Tg= Triglycerides; TC=Total Cholesterol; eGFR= Estimated Glomerular Filtration Rate; ECG= Electrocardiogram

Figure 1: Associated co- morbidities in the study population

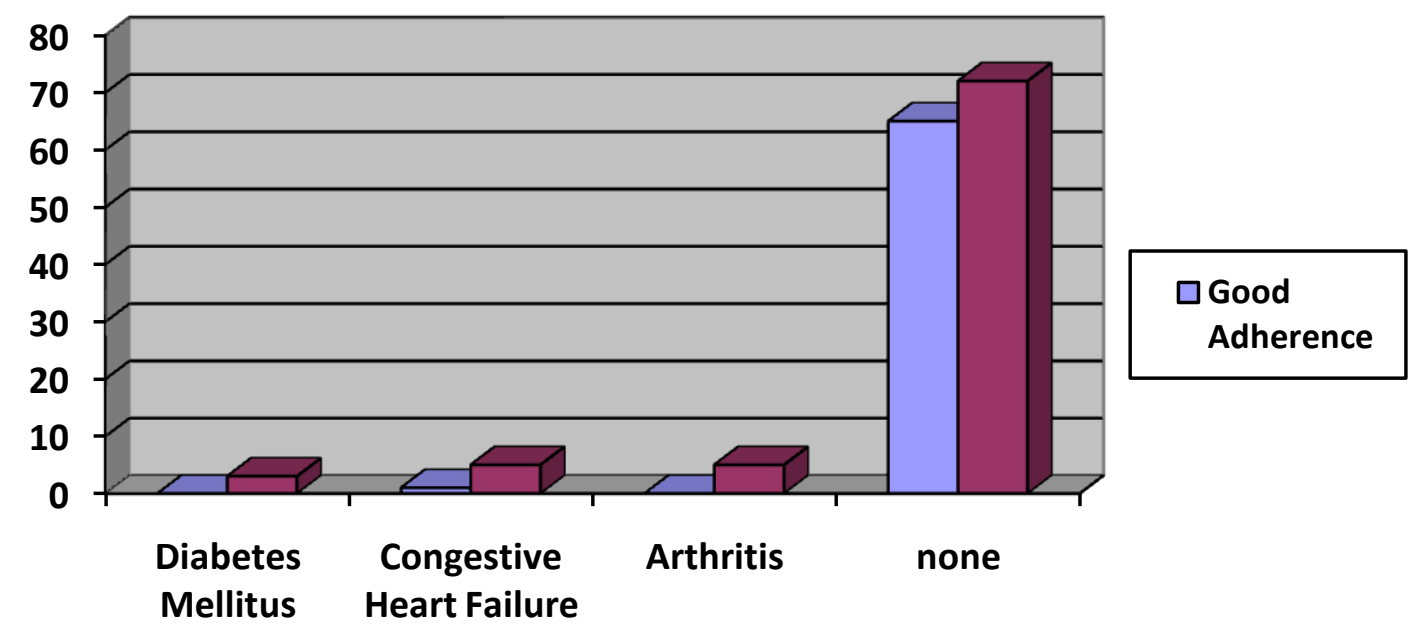

Figure 2: Pattern of Blood Pressure Targets among the Study Population

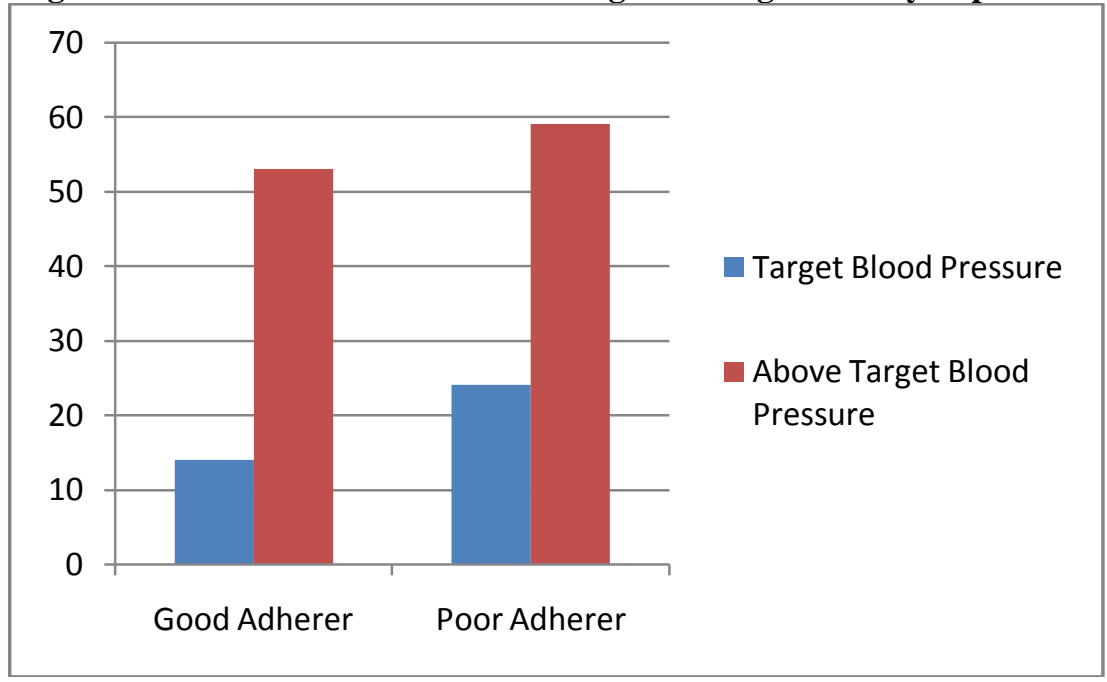

(OR, 0.65; 95\% CI, 0.31 to $1.38 ; \mathrm{p}=0.26)$ 